\title{
【研究简报】
}

\section{Fuzzy 格上的点式一致结构与点式度量}

\author{
史福贵 \\ (烟台师范学院数学系, 姆台 264025)
}

\section{关链词 Fuzzy 格 点式一致结构 点式度量 完全正则性 远城映射族}

关于格拓扑学中的一致结构与度量理论已有许多引人注目且有创造性的工作(如文献 [1 6]及文献 [4]中的相应文献). 但它们多是 Hutton 与 Erceg 无点派工作的继续和推广, 不 能直接反映格上点式拓扑的特点. 本文的目的就是在 Fuzzy 格上建立一种点式一致结构与点 式度量理论。

本文 $L$ 恒为 Fuzzy 格, $M$ 表示 $L$ 中所有分子之集, $L$ 也常记为 $L(M)$. 对空集 $\emptyset \subset L$, 规定 $\wedge \emptyset=1, \vee \emptyset=0$. 有关正则与正规性的定义见文献 [7]. 其他未声明的概念与符号均源 于文献 $[8]$.

设 $L(M)$ 是 Fuzzy 格, 两表示 $M$ 到 $L$ 的满足条件: $\forall x \in M, x \neq f(x)$ 的全体映射.

多中的一个保序映射 $f$ 称为对称的, 如果 $\forall x, y \in M$, 存在分子 $a \neq x^{\prime}$ 使 $y \neq f(a) \Rightarrow$ 存 在分子 $b$ 集 $y^{\prime}$ 使 $x$ 䒠 $f(b)$.

定义 $1 L$ 上的一个点式拟一致结构 ${ }^{[9]}$ 称为点式一致结构, 如果其有一个由对称元组成 的基.

定理 1 设 4 是 $L(M)$ 上的点式一致结构, $\forall A \in L$, 规定

$$
c(A)=V: a \in M \mid \forall f \in \Psi, A \neq f(a): \text {, }
$$

则

(1) $\forall a \in M, a \leqslant c(A) \Leftrightarrow \forall f \in \mathcal{M}, A \neq f(a)$;

(2) $c: L \rightarrow L$ 是闭包算子, 从而在 $L$ 上可导出一个余拓扑, 记为 $\eta(\vartheta)$.

定义 2 设 $\eta$ 是 $L(M)$ 上的余拓扑, $(I, \eta)$ 称为完全正则的, 如果 $\forall x \in M, \forall B \in \eta$, 当 $x \geqslant B$ 时, 存在一族元: $A(t)\} t \in(0,1) \mid$ 使当 $s<r$ 时, $x \geqslant A(s) \geqslant A(s)^{\circ} \geqslant A(r)^{-} \geqslant A(r)$ $\geqslant B$.

定理 $2(L(M), \eta)$ 是完全正则的当且仅当 $\forall x \in M, \forall B \in \eta$, 当 $x B$ 时, 存在递减 映射 $F:(0,1) \rightarrow L$ 使 $x \neq F(0+), B \leqslant F(1-)$ 且 $\forall t \in[0,1], F(t-) \in \eta, F(t+) \in \eta^{\prime}$.

定理 $3(L(M), \eta)$ 可点式一致化(即存在 $L$ 上的点式一致结构 $\psi$, 使 $\eta=\eta(\lambda)$ ) 当且仅 当其是完全正则的.

定理 $4 L F$ 单位区间 $[0,1](L)$ 可点式一致化.

定义 3 映射 $d: M \times M \rightarrow[0,+\infty)$ 称为 $L(M)$ 上的点式 $p$. 度量, 如果 $d$ 满足下面 (M1) (M4).

(M1) $\forall a \in M, d(a, a)=0$;

(M2) $\forall a, b, c \in M, d(a, c) \leqslant d(a, b)+d(b, c)$;

(M3) $\forall a, b \in M, d(a, b)=\Lambda d(a, c)$, 这里 $c \ll b$ 指的是 $c \in \beta^{*}(b)$; 
(M4) (i ) $\forall a, b, c \in M, a \leqslant b \Rightarrow d(a, c) \leqslant d(b, c)$,

(ii) $\forall x, y \in M, \exists a$ 毛使 $d(a, y)<r \Rightarrow \exists b$ 䒠的使 $d(b, x)<r$.

点式 $p$. 度量 $d$ 称为点式度量, 如果 $d$ 满足下面

(M5) $\forall a, b \in M, d(a, b)=0 \Rightarrow a \leqslant b$.

定理 $5 L(M)$ 上的点式 $p$. 度量 $d$ 的远域映射族 ${ }^{[9]}$ 恰满足下面

(R1) $\forall a \in M, \forall r>0, a$ 䒠 $P_{r}(a)$;

(R2) $\forall r, s>0, P_{r} \odot P_{s} \geqslant P_{r+s}$;

(R3) $\forall a \in M, \forall r>0, P_{r}(a)=\bigwedge_{s<r} P_{s}(a)$;

(R4) $\forall a \in M, \wedge_{r>0} P_{r}(a)=0$;

(R5) $\forall r>0, P_{r}$ 是对称的.

定理 6 设映射族 $\left\{P_{r} \mid P_{r}: M \rightarrow L, r>0\right\}$ 满足 $(\mathrm{R} 1) \sim(\mathrm{R} 5), \forall(a, b) \in M \times M$, 规定 $d(a, b)=\Lambda\left\{r \mid b \$ P_{r}(a)\right\}$,

则 $d$ 是 $L$ 上的点式 $p$. 度量且 $\left.\left|P_{r}\right| r>0\right\}$ 恰是 $d$ 的远域映射族.

定理 7 Fuzzy 格上的点式 $p$. 度量的远域映射族为某点式一致结构的基, 称此点式一致 结构为此点式 $p$. 度量所诱导的.

定理 8 Fuzzy 格上的点式 $p$. 度量是点式度量当且仅当其导出的余拓扑是 $T_{1}$ 的, 这里 点式 $p$. 度量导出的余拓扑是指其诱导的点式一致结构导出的余拓扑.

定理 9 Fuzzy 格上的点式一致结构可由点式 $p$. 度量所诱导 (也称可点式 $p$. 度量化) 当 且仅当其有可数基.

推论 $L F$ 单位区间可点式 $p$. 度量化.

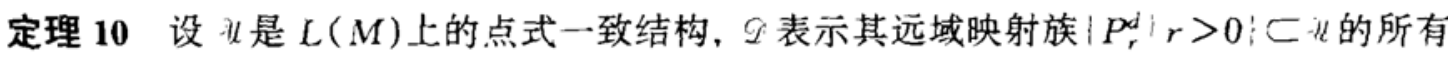
点式 $p$. 度量 $d$ 所成的集,则 $\cup i P_{r}^{d} ; r>0, d \in \mathscr{D} ;$ 是 $\lambda$ 的子基.

引理 1 设 $\eta$ 是 $L$ 上的余拓扑, 则下列条件等价:

(1) $(L, \eta)$ 是正规的;

(2) $\forall A, B \in \eta$, 当 $A \leqslant B^{\prime}$ 时, 存在可数开元族 $\left\{H_{i} \mid i \in j !\right\}$ 使 $A \leqslant \vee_{i \in} H_{i}$ 且 $\forall i \in i$, $H_{i}^{-} \leqslant B^{\prime}$;

(3) $\forall A, B \in \eta$, 当 $A \leqslant B^{\prime}$ 时, 存在一族元 $\left\{H_{t}\right\} t \in(0,1)$; 使 $\forall s, t \in(0,1)$, 当 $s<t$ 时, $A \leqslant H_{t} \leqslant H_{t}^{-} \leqslant H_{s}^{o} \leqslant H_{s} \leqslant B^{\prime}$;

(4) $\forall A, B \in \eta$, 当 $A \leqslant B^{\prime}$ 时, 存在递减映射 $F:(0,1) \rightarrow L$, 使 $A \leqslant F(1-) \leqslant F(0+)$ $\leqslant B^{\prime}$ 且 $\forall t \in[0,1], F(t-) \in \eta, F(t+) \in \eta^{\prime}$.

引理 2 若 $(L, \eta)$ 是正则 $C_{\text {II }}$ 的, 则必是正规的.

定理 11 设 $(L, \eta)$ 是 $C_{\text {II }}$ 的, 则 $(L, \eta)$ 可点式 $p$. 度量化当且仅当其是正则的; $(L, \eta)$ 可点式度量化当且仅当其是正则 $T_{1}$ 的.

致谢四沁联合大学刘应明老河对售者给予了热情鼓励，在此深表㴬意。本工作为山东省自 然科学基金资助项目。

\section{参考文献}

1 Hutton B. Uniformities on fuzzy topological spaces. J Math Anal Appl, 1977, 58:559 


\section{【研究简报】}

\section{多项式实零点个数的新判据}

$$
\text { 林 亮(1) 刘一我 (2) }
$$

(1)桂林工学院基础部, 生林 541004; (2)中南工业大学数学系, 长沙 410083)

\section{关链词多项式 实点}

众所周知, 对一般的 $n$ 次实系数多项式 $f(x)$, 如何判定它的实零点个数, 这一问题是多项式理 论中的一个重要组成部分, 在数学、力学、物理学等许多方面都有重要应用. Sturm(参见文献 [1] 第 六章) 虽然指出了用Euclid 辗转相除法解决上述问题的途径, 但未能直接给出 $f(x)$ 的系数与其实零 点个数之间的关系, 故不便于直接应用, 更不便于对上述问题作进一步的理论分析. 类似于 Hurwitz(参见文献 [2] 附录 5) 用一些行列式的正、负符号成功地给出 $f(x)$ 的零点都有负实部的充 要条件, 本文在 $f^{\prime}(x)$ 与 $f(x)$ 的结式中取 $n$ 个子行列式作为判别式, 通过这些判别式的正、负符号 给出判定 $f(x)$ 的实零点个数的公式, 从而解决了上述问题. 设

$$
\begin{aligned}
& f(x)=a_{n} x^{n}+a_{n-1} x^{n-1}+\cdots+a_{0}, \\
& g(x)=b_{m} x^{m}+b_{m-1} x^{m-1}+\cdots b_{0},
\end{aligned}
$$

其中 $a_{n} b_{m} \neq 0, n \geqslant m$. 记

$$
D_{0}(g, f)=b_{m}^{n-m} .
$$

$\forall k \in\{1,2, \cdots, m !$, 在 $g$ 与 $f$ 的结式

$$
D(g, f)=\left|\begin{array}{ccccccccc}
b_{m} & b_{m-1} & \cdots & \cdots & b_{0} & & & \\
& b_{m} & b_{m-1} & \cdots & \cdots & b_{0} & & \\
& & \cdots & \cdots & \cdots & \cdots & \cdots & & \\
& & & \cdots & \cdots & \cdots & \cdots & \cdots & \\
& & & & b_{m} & b_{m-1} & \cdots & \cdots & b_{0} \\
a_{n} & a_{n-1} & \cdots & \cdots & \cdots & a_{0} & & & \\
& a_{n} & a_{n} & \cdots & \cdots & \cdots & a_{0} & & \\
& & \cdots & \cdots & \cdots & \cdots & \cdots & \cdots & \\
& & & a_{n} & a_{n-1} & \cdots & \cdots & \cdots & a_{0}
\end{array}\right|
$$

\footnotetext{
Erceg M A. Metric in fuzzy set theory. J Math Anal Appl, 1979,69:205

刘应明. 不分明拓扑空间中完全正则性的点式刻划与的入定理. 中国科学, $A$ 转, 1982, (8):675

刘应明、梁基华. Fuzzy 招扑学—层次结构和点式处理. 数学进展, 1994, 23(4):304

梁基华。完全分配格上的一致结构. 科学通报, 1989, 34(14):1049

彭育威. 格上点式 $p . q$. 度量理论的若干进展. 四川大学学报(自然科学版)，1990，27(3):275

Hutton B, Reilly I. Separation axioms in fuzzy topological spaces. Fuzzy Sets and Systems, 1980, 3:93

王国俊．完全分配格上的点式拓扑．陕西师大学报(自然科学版)，1985、13(1):1;13(2):1

史楅贵. 完全分配格上的点式拟一致结构与 $p . q$. 度睡. 数学学报, 1996, 39(5):701
} 